\title{
AN ASSESSMENT OF SHADOW ENHANCED URBAN REMOTE SENSING IMAGERY OF A COMPLEX CITY - HONG KONG
}

\author{
Cheuk-Yan Wan*, Bruce A. King, Zhilin Li \\ The Department of Land Surveying and Geo-Informatics, The Hong Kong Polytechnic University \\ Hung Hom, Kowloon, Hong Kong - (10900974R, 1sbaking, 1szlli)@polyu.edu.hk
}

\section{Youth Forum}

KEY WORDS: Shadow detection, Image enhancement, Shadow removal, Quickbird, Urban, Remote Sensing

\begin{abstract}
:
Large portions of shadowed areas in satellite images of urban areas can affect the accuracy of classification and thus reduce an image's effectiveness in urban remote sensing applications. This is particularly acute in cities such as Hong Kong where dense high-rise buildings cast many long shadows across a variety of different surface types. One solution to this problem is to enhance shadowed areas so their spectral range becomes closer to their corresponding non-shadowed areas. Shadowed areas were automatically selected and two techniques, Gamma correction and Linear Correlation Correction, were applied to three study sites of a $2.4 \mathrm{~m}$ Quickbird image. The selected study sites represent typical urban types of Hong Kong, ranging from high-rise commercial to low-rise residential areas. The shadow detection algorithm is based on the spectral shape index and its limitation is discussed. The histograms of the corresponding non-shadowed areas, the original and the enhanced shadow areas are used to compare the spectral range. The results show that the enhanced areas, in band ratios such as NDVI, show greater similarity after enhancement, but they also look darker than the non-shadowed areas. Where continuous shadowed areas such as in commercial areas, the spectral range cannot be restored.
\end{abstract}

\section{INTRODUCTION}

\subsection{Background}

Hong Kong is a famous vibrant city with many high-rise buildings. With limited land supply, residential and commercial buildings are usually built with more than 30 storeys. The impact of such development on day-time high-resolution satellite images is that shadows are unavoidable and seriously affect the image quality. High building density and heights cause large portions of land surfaces to be occluded or under shadow. Image classification and interpretation is thus a challenging task.

Shadow enhancement is a process to compensate the brightness difference between shadow and non-shadow areas. Although there are successful cases in different countries, an application in Hong Kong has to deal with water bodies, asphalt surfaces and even shadows on water.

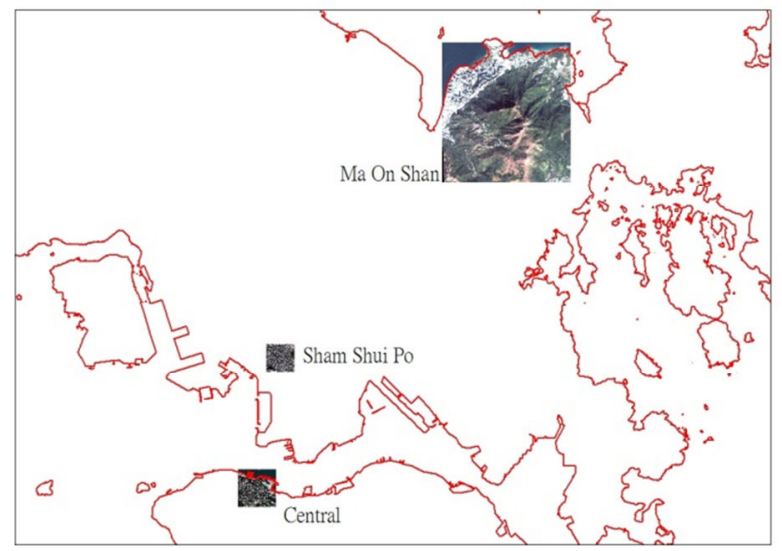

Figure 1. Overview map of the study areas

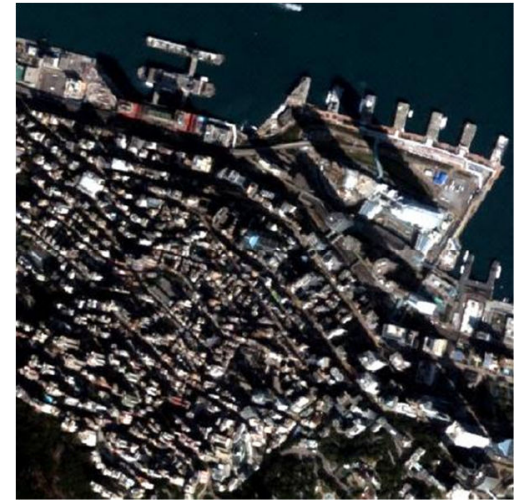

Figure 2. Typical urban area in Hong Kong-Central (Quickbird image)

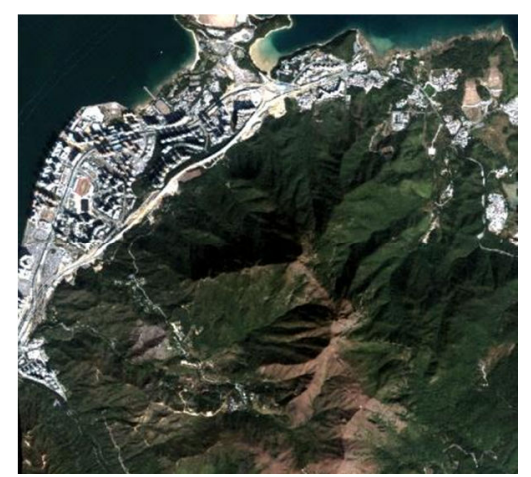

Figure 3. Ma On Shan (Quickbird image)

\footnotetext{
* Corresponding author.
} 


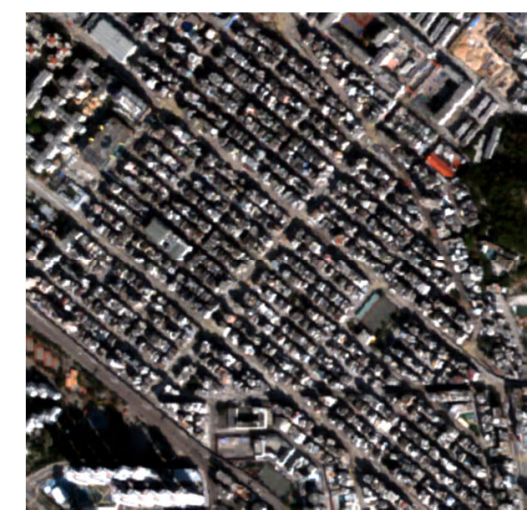

Figure 4. Sham Shui Po (Quickbird image)

The results of applying gamma correction and linear correlation correction are examined in three selected areas in Hong Kong. The study areas consist of different environments: shadows from densely distributed buildings; shadows from mountainous terrain; and areas that are close to water bodies. The selected areas are located across the territory of Hong Kong (Figure 1) and can be seen in Figure 2 (Central), Figure 3 (Ma On Shan), and Figure 4 (Sham Shui Po).

\subsection{Negative effects of shadows}

Due to the darker pixels, feature identification becomes a problem when the same surface having large color differences. The negative effect of shadows including radiometric information loss (Arevalo et al., 2006; Chen et al., 2007) and inaccurate change detection results due to different azimuth angle (Yamazaki et al., 2009). In order to minimize the negative effect of shadows in aerial photos and satellite images, the concept of brightness compensation was introduced (Shu and Freeman, 1990). This process requires shadows firstly be detected and extracted, and then different algorithms applied to enhance these shadow regions.

\subsection{Related Works}

1.3.1 Shadow detection: Shadow detection techniques can be categorized as properties based and model based. For properties based detection, Cai et al. (2010) suggested 3 different indices based on HSI color space which is more accurate than using HSI color space only. Sarabandi et al. (2004) and Arevalo et al. (2006) made use of the $\mathrm{C}_{3}$ layer from color invariant indices $\mathrm{C}_{1} \mathrm{C}_{2} \mathrm{C}_{3}$ for shadow detection. Texture filter and region growing techniques are then applied to extract shadows. Tsai (2006) compared the effectiveness of different invariant color spaces for shadow detection, including HSI, $\mathrm{HSV}, \mathrm{HCV}, \mathrm{YIQ}$ and $\mathrm{YC}_{\mathrm{b}} \mathrm{C}_{\mathrm{r}}$ models. He concluded that HSI, YIQ and $\mathrm{YC}_{\mathrm{b}} \mathrm{C}_{\mathrm{r}}$ are the most suitable color space for shadow detection. However, when these algorithms were applied to Hong Kong urban area, shadows are usually mixed with water bodies which may get wrongly enhanced. In Chen et al. (2007), 5 empirical indices were suggested to separate shadows from water and is named as Spectral Shape Index which is able to deal with the scenario in Hong Kong.

Model based shadow detection algorithms require a digital building model DBM together with digital surface model DSM (Zhou, 2005). Nakajima et al. (2002) made use of Airborne Laser Scanning data and DSM for shadow simulation in urban area. However, a high accuracy DSM is required to produce fair result. In Hong Kong, an accurate DSM can hardly be obtained due to the fact that high-rise buildings are densely distributed on mountainous terrain.

1.3.2 Shadow Enhancement: Here, existing algorithms include histogram matching, gamma correction or linear-correlation correction.

Histogram matching works by correcting the brightness distribution of two given images as similar as possible (Sarabandi et al., 2004). Dare (2005) comment that the algorithm only works well while the histogram information of nearby regions is used. Zheng \& Wang (2008) reported that it is difficult to extract histogram information of the same type of surfaces.

Gamma correction treats shadow as a source of noise that the brightness of the pixels is being affected (Sarabandi et al., 2004). The Gamma parameter is estimated based on the mean pixel values of shadowed and non-shadowed areas. Massalabi et al. (2004) reported that the precision of the land-use map created increases when a Gamma Corrected image is used. The equation of gamma correction is described in equation (1) (Zhan et al., 2005).

$$
D N_{\text {output }}=2047 \times\left(D N_{\text {input }} \div 2047\right)^{\frac{1}{\gamma}}
$$

Where the $\mathrm{DN}_{\text {input }}$ refers to the pixel values under shadow and the $\mathrm{DN}_{\text {output }}$ is the non-shadow pixel values nearby. The gamma value $\gamma$ has to be determined for each image using local sampling data.

According to Sarabandi et al. (2004), if shadow is treated as a combination of additive and multiplicative noise, the brightness of shadows can be enhanced by a linear function. The linear function can be obtained from a number of pairs of pixel values (under sunlight and shadow) (Yamazaki et al., 2009). Among the three existing enhancement algorithms, both Sarabandi et al. (2004) and Zheng \& Wang (2008) agreed that linear correlation correction gave better results after enhancement. The color tone is much closer to its surrounding afterwards.

\section{RESEARCH METHODOLOGY}

\subsection{Shadow detection algorithm}

For shadow detection, properties based algorithm is being used, this is due to the fact that an accurate DSM and DBM is not available in Hong Kong. The existing digital terrain model DTM for the whole Hong Kong is combined by several techniques and measurement, the accuracy of this DTM varies from location to location.

Shadow detection based on HSI transformation, $\mathrm{C}_{1} \mathrm{C}_{2} \mathrm{C}_{3}$ color space and the empirical indices suggested by Chen et al. (2007) has been examined. It is found that HSI transformation is not able to classify dark surfaces that are not under shadow, and $\mathrm{C}_{3}$ layer is quite noisy and water features are easily mixed with shadows. For the 5 indices suggested in Chen et al. (2007), the fifth index is recommended and named as Spectral Shape Index (SSI). However, when it is applied to Hong Kong, quite a number of non-shadow features are being extracted, the index suggested is described in equation (2). 


$$
\text { SSI }=1 \times \text { Red }+ \text { Blue }-2 \times(\text { Green })
$$

After examined the shadow detection results from the rest 4 indices, it is found that the second index separate shadows from water much better than the index recommended. The index used in this study is described in equation (3)

$$
S S I=(\text { Green }- \text { Red }) \div(\text { Red }+N I R)
$$

The threshold value for each image has to be determined for each image in order to create the shadow images.

\subsection{Shadow enhancement algorithm}

In order to enhance each spectral layer individually, a shadow mask has to be applied to all spectral layers. In this study, the enhancement result by Gamma correction and Linear-correlation correction is compared. The pixel values of shadow areas and its adjacent non-shadow areas are used to determine the Gamma parameter using equation (1).

For Linear-correlation correction, a linear regression model is required for each spectral layer. In each image, a number of shadow and non-shadow pairs of areas (same type of surfaces) are selected to generate the regression model. The average pixel values of these equal size areas ( 4 pixel x 4 pixel) is being recorded for creating the linear regression model. Compared to just using pair of points for creating the regression model, using equal size areas can minimized the effect that extreme pixels are involved.

No matter which algorithm is applied, after the shadowed parts of the images have been enhanced, the non-shadow parts have to be merged to create the enhanced image. Merging was achieved by applying the shadow mask to the enhanced image (only shadows are enhanced) so as to make the non-shadow areas within become 0 . Once this is done, the enhanced image can be overlaid with the original image with shadowed areas set as value 0 . The shadow enhanced regions will then replace the original shadow regions.

The objective of this study is to examine the shadow enhancement result under extreme urban structure. NDVI is used to simulate the results of urban vegetation detection before and after enhancement is carried out.

\section{CASE STUDIES}

\subsection{Characteristic of selected study areas}

To examine the shadow enhancement result under extreme environment such as those typical to Hong Kong, 3 areas are selected which cover most of the complicated scenarios.

Central - located in the north side of Hong Kong Island, having a mountainous terrain from the Peak to the sea shore. Central is the CBD in Hong Kong, tall residential buildings are mainly distributed from mountainside to the top, while even taller commercial buildings are distributed close to the coastline. Buildings in this district can be built up to 50 or even 60 storeys, road surfaces can hardly be seen from satellite images. Shadows in Central are mainly from buildings.
Ma On Shan - located in the north east of Hong Kong, is a newly developed town with lower building density. It is beside a country park and close to natural coast. Not many buildings in this district are tall, some of them are around 30-40 storeys while most of the others are lower. Shadows in Ma On Shan are from buildings and mountainous terrain within the country park.

Sham Shui Po - located in the center of the Kowloon Peninsula of Hong Kong, is an old developed area with buildings densely distributed, and roads are narrow. Sham Shui Po is not close to the coastline and the terrain is rather flat. Most of the buildings in Sham Shui Po are around 10 storeys and are well organized into regular building blocks. Shadows formed in this district mainly by buildings.

\subsection{Shadow detection result}

The shadow extraction result is shown in this section. Figure 5 shows the shadow mask for Central, huge amount of shadows can be seen (areas in white). Compared to Figure 2, most of the water bodies are not being misclassified as shadows except the case that shadows fall onto the water surfaces.

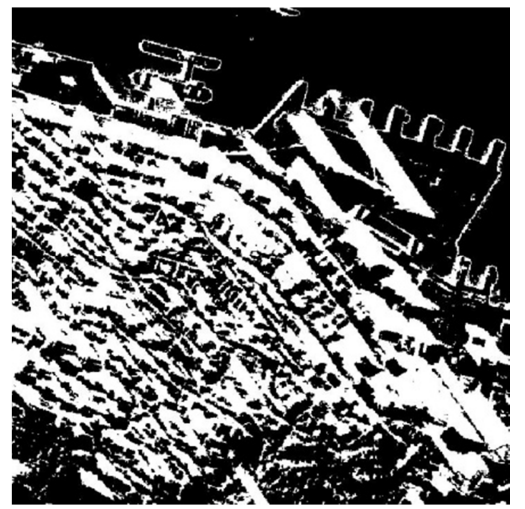

Figure 5. Central - shadow extraction result (shadows in white)

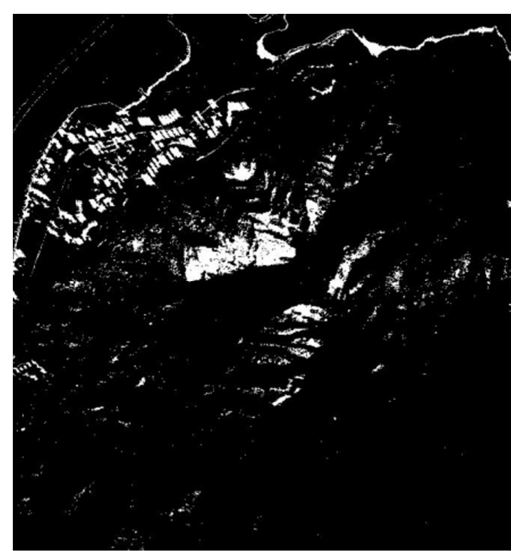

Figure 6. Ma On Shan - shadow extraction result

For Ma On Shan, shadows by mountainous areas and buildings are extracted. However, the natural coast areas are being misclassified as shadows. These areas are in fact, shallow water (shown in Figure 3). 


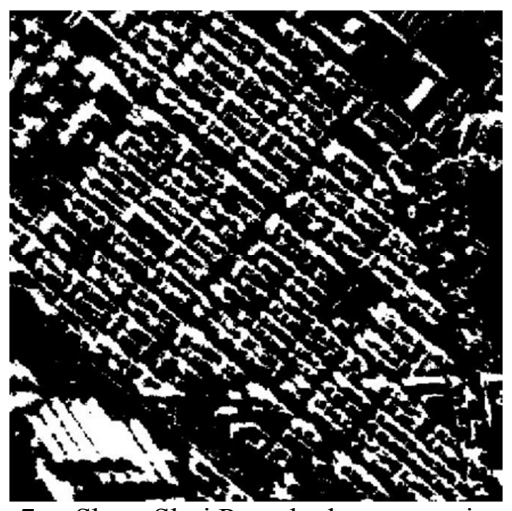

Figure 7. Sham Shui Po - shadow extraction result

Shadows extracted in Sham Shui Po district are mainly formed by buildings. Except a larger shadow is found with in the lower left corner, rest of the shadows are small in size.

Compared the shadow extraction result among 3 selected locations, the algorithm used is not able to deal with shallow water being misclassified. For shadows fall onto water surfaces, the shadow mask created is quite noisy and only parts of them are being extracted.

\subsection{Shadow enhancement result}

3.3.1 Central: 16 pairs of test areas were used to establish the linear regression model for all four spectral bands. The enhancement result is shown in Figure 8.
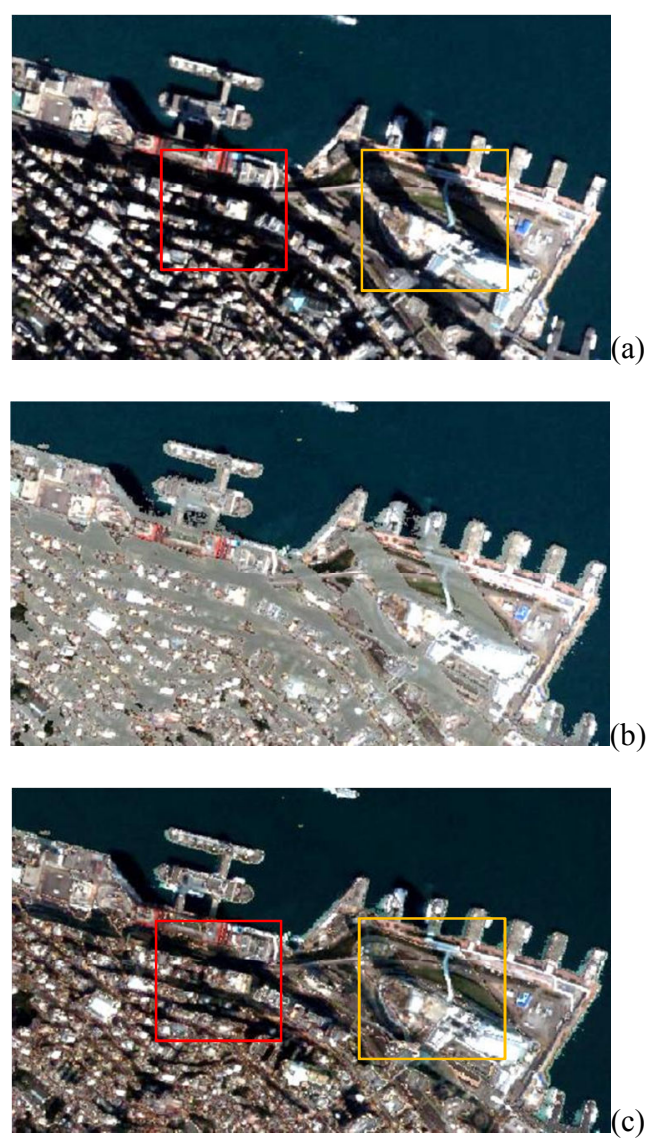

Figure 8. Central - a) Original image; b) By Gamma Correction; c) By Linear-Correlation Correction

Compared the enhancement result, Gamma Correction (Figure 8b) cannot provide better appearances. This is due to the fact that one gamma parameter is used for the entire image; the image should be further divided into smaller parts to estimate the local gamma parameters. Enhancement by the linear regression model gives a much better result; the tones for most of the shadow enhanced regions are much closer to its surrounding.

However, it is also found that some of the shadowed regions did not improve much after linear enhancement; this is shown in the red box on the left hand side of Figure 8a and 8c. After checking the pixel values of these areas, they are almost the darkest pixels within all layers having rather small standard deviation. When compared to the result of the yellow box on the right, roads, flyover and the grassland appear much closer to its surrounding.

3.3.2 Ma On Shan: A total 18 pairs of testing areas were selected for establishing the linear regression model of Ma On Shan image. A similar enhancement result is obtained comparing to Central, result as shown in Figure 9.
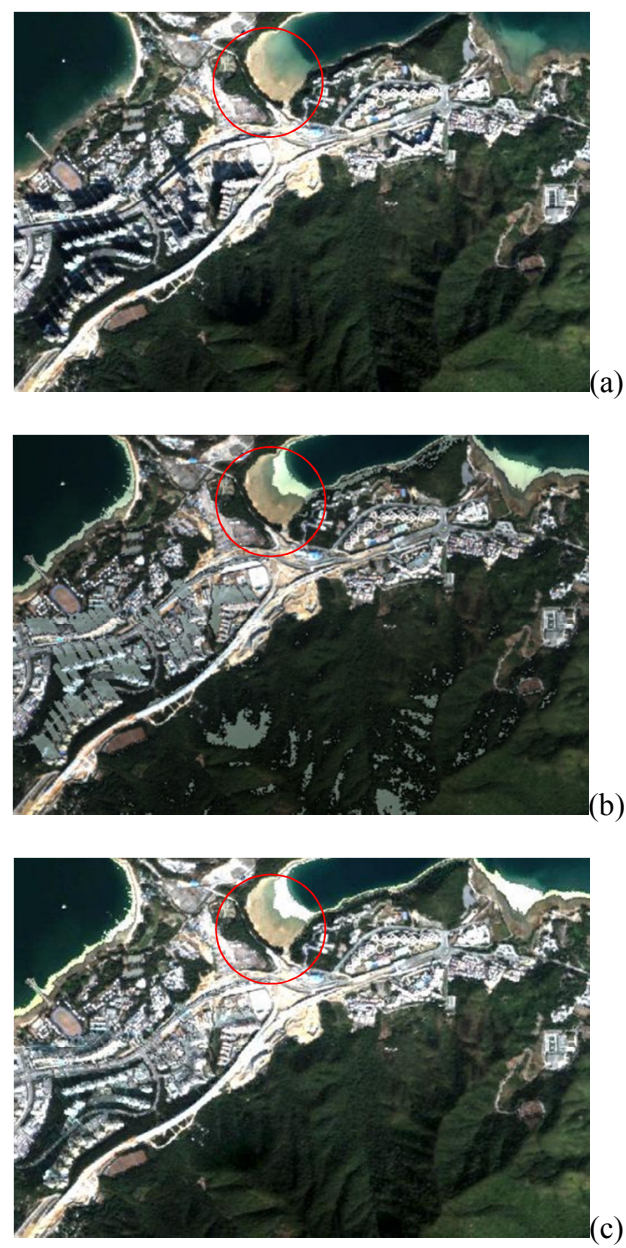

Figure 9. Ma On Shan - a) Original image; b) By Gamma Correction; c) By Linear-Correlation Correction

Similar performance is obtained comparing the result between Central and Ma On Shan, Linear-Correlation Correction gives better appearance after enhancement. In Figure 9c, the road on the left can now be seen as one object, different from Figure 9a that the road is being "separated" by shadows. The enhancement result is generally acceptable except the natural coast is being wrongly extracted and enhanced. 
3.3.3 Sham Shui Po: The linear regression model for Sham Shui Po is generated based on 17 pairs on testing areas. The enhancement result is shown in Figure 10.

Despite the result showing better shadow enhanced image using linear regression, shadow at the lower left corner of Figure 10c also shows that even if linear regression is used, the shadow enhanced region is still slightly darker than its surrounding.
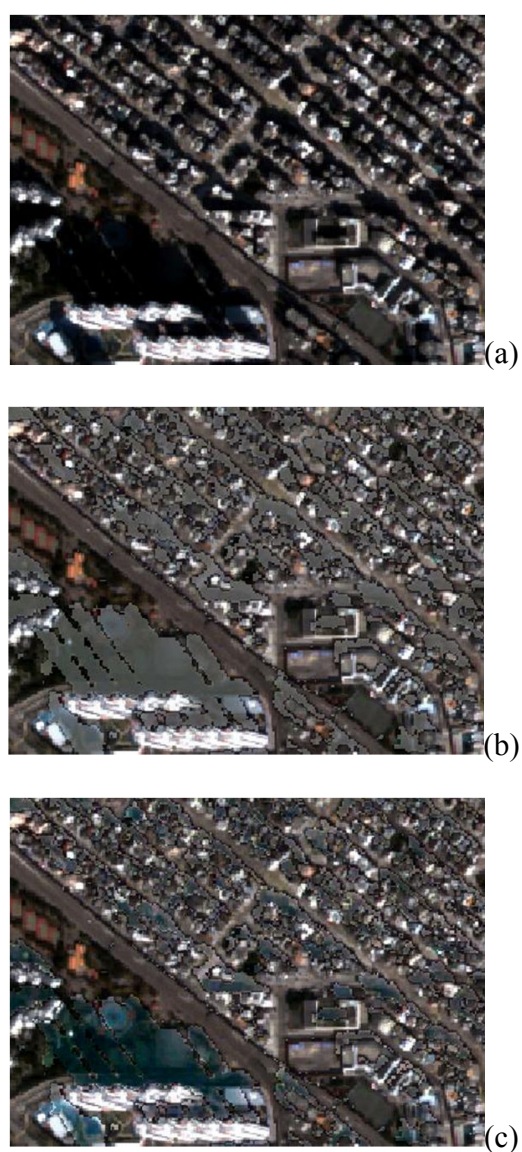

Figure 10. Sham Shui Po - a) Original image; b) By Gamma Correction; c) By Linear-Correlation Correction

\subsection{Using shadow enhanced image for vegetation mapping}

In this section, Normalized Difference Vegetation Index (NDVI) is used to extract land cover of vegetation after shadow enhancement. Figure 11 shows the NDVI image of Central before and after shadow enhanced.

From Figure 11a and 11b, it could be seen that vegetation under shadow is able to be detected after enhancement. However, for some of the regions, due to the rescaling of enhanced spectral layers, the NDVI is not correctly calculated. Similar result is obtained for Ma On Shan image (Figure 12a and $12 \mathrm{~b}$ ), vegetation under shadow can be extracted, however, the NDVI values differs quite a lot compared to its neighbor region. Most of these areas are shadows having relatively low pixel values, and these low pixel values are not likely to be significantly enhanced under a linear regression model.
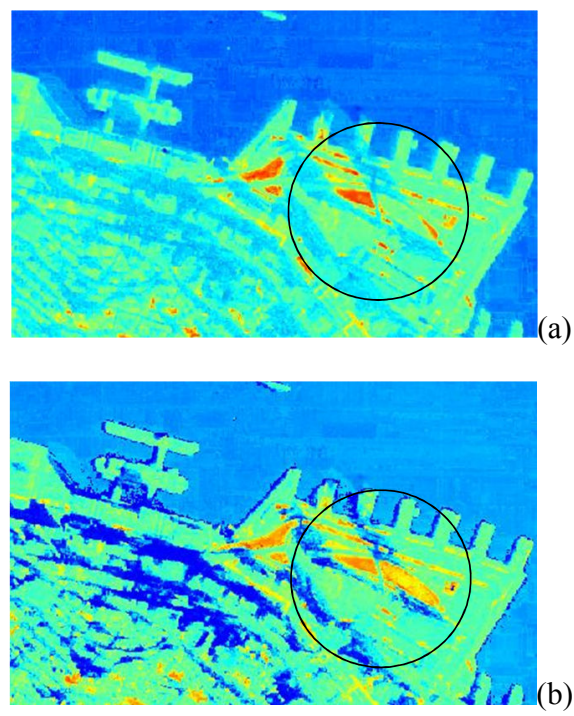

Figure 11. NDVI of Central - a) Original image; b) By Linear-Correlation Correction

When comparing the black circle between Figure 12a, 12b and 12c, Gamma Correction did not show any significant improvement in detecting vegetation. NDVI by Linear-Correlation Correction is able to detect it as vegetation, but the value is much higher than its surrounding. To minimize this effect, the rescale process in both red band and infrared band have to be redesigned.
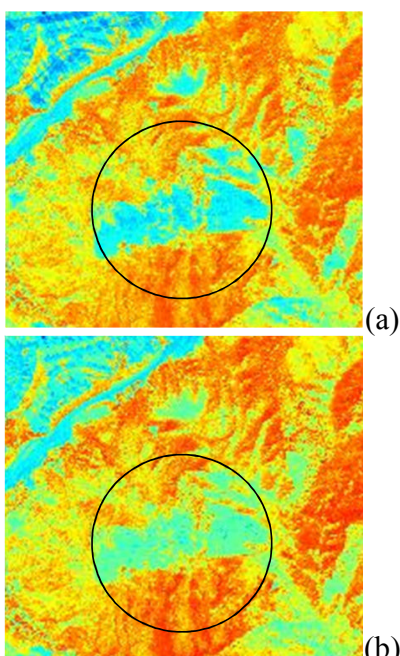

a)

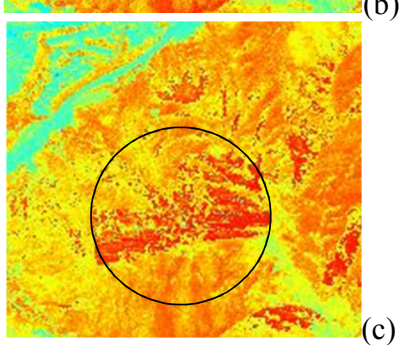

Figure 12. NDVI of Ma On Shan - a) Original image; b) By Gamma Correction; c) By Linear-Correlation Correction

\subsection{Problems associated with shadow enhancement}

For Gamma Correction, it is not appropriate to apply one single gamma value for the whole image and it would be much better when the image is further divided into smaller parts. However, when shadows are large and a number of different 
types of surfaces are being involved, finding a gamma values for each type of land cover is difficult. This also involves the process of classifying shadows of different land covers.

Compared to Gamma Correction, Linear-Correlation Correction gives better result in terms of appearances. However, using a linear model may not be able to enhance shadows that are extremely dark in certain spectral bands. This effect is serious when sunlight (or any light sources including scattering) is being blocked like in Central district (Figure 8). Despite of the issue, some of the shadows may not be "enhanced" at all. Since linear regression model is used, it is possible that some of the pixel values decreased afterwards or exceed the level of 0-2047 (11bit image). The maximum and minimum pixel value is now set to 2047 and 0 . However, in general, it is not likely to have pixel values of 0 and 2047 . Rescaling process has to be redesigned in order to correct the data range, so that when calculating ratios like NDVI, the resulted value is within normal range (from -1 to +1 for NDVI).

\subsection{Future work}

In the future, the histograms of enhanced regions and their neighboring non-shadow regions will be compared. A more systematic spectral fidelity assessment will be carried out. Despite of the spectral fidelity assessment, shadow enhancement using amount conversion of statistic (Nakajima et al., 2002) may also be compared. This algorithm mainly made use of the average and standard deviation of pixel values of shadow and non-shadow pairs.

\section{CONCLUSION}

Shadow detection using the spectral shape index is able to deal with the issues that water and shadows being misclassified. However, it will have some problems when there is natural coast and shadows fall onto the water. Gamma Correction and Linear-Correlation Correction is applied to 3 selected areas in Hong Kong. Results showing that shadow enhanced image by Linear-Correlation Correction has better appearances and its NDVI image is able to extract vegetation that are originally under shadow.

This study shows that even linear regression model is applied to complicated urban structure, when sunlight is being blocked and no scattering from other light sources, shadows can hardly be enhanced significantly.

\section{ACKNOWLEDGEMENT}

The work described in this paper was supported by the grants from the Hong Kong Polytechnic University. The authors would like to thank Remote Sensing Research Group of the Department of Land Surveying and Geo-Informatics for providing the Quickbird images.

\section{REFERENCES}

Arevalo, V., Gonzalez, J., Valdes, J., \& Ambrosio, G., 2006. Detecting Shadows in QUICKBIRD Satellite Images. ISPRS Commission VII Mid-term Symposium "Remote Sensing: From Pixels to Processes", (pp. 330-335). Enschede, the Netherlands.
Cai, D., Li, M., Bao, Z., Chen, Z., Wei, W., \& Zhang, H., 2010. Study on Shadow Detection Method on High Resolution Remote Sensing Image Based on HIS Space Transformation and NDVI Index. 18th International Conference on Geoinformatics, 2010 (pp. 1-4). Beijing: IEEE.

Chen, Y., Wen, D., Jing, L., \& Shi, P., 2007. Shadow information recovery in urban areas from very high resolution satellite imagery. International Journal of Remote Sensing, 28(15), pp. 3249-3254.

Dare, P. M., 2005. Shadow Analysis in High-Resolution Satellite Imagery of Urban Areas. Photogrammetric Engineering \& Remote Sensing, 17(2), pp. 169-177.

Nakajima, T., Tao, G., \& Yasuoka, Y., 2002. Simulated recovery of information in shadow areas on IKONOS image by combing ALS data. The 23th Asian Association of Remote Sensing (AARS) 2002. Kathmandu, Nepal.

Sarabandi, P., Yamazaki, F., Matsuoka, M., \& Kiremidjian, A., 2004. Shadow Detection and Radiometric Restoration in Satellite High Resolution Images. IEEE IGARSS '04., 6, pp. 3744-3747.

Shu, J., \& Freeman, H., 1990. Cloud shadow removal from aerial photographs. Pattern Recognition, 23(6), pp. 647-656.

Tsai, J. D., 2006. A Comparative Study on Shadow Compensation of Color Aerial Images in Invariant Color Models. IEEE Transactions on Geoscience and Remote Sensing, 44(6), pp. 1661-1671.

Yamazaki, F., Liu, W., \& Takasaki, M., 2009. Characteristic of shadow and removal of its effects for remote sensing imagery. IEEE IGARSS '09., 4, pp. 426-429.

Zhan, Q., Shi, W., \& Xiao, Y., 2005. Quantitative analysis of shadow effects in high-resolution images of urban areas. $W G$ VIII/1 Joint Symposia URBAN - URS 2005. Tempe, AZ, USA: ISPRS.

Zheng, Q.-y., \& Wang, Q.-m., 2008. Shadow restoration method of Quickbird satellite remotely sensed imagery. Computer Engineering and Application, 44(17), pp. 30-32.

Zhou, G., 2005. Urban Large-scale Orthoimage Standard for National Orthophoto Program. IEEE Geoscience and Remote Sensing Symposium, 2005. IGARSS '05. Proceedings. (pp. 1214-1217). 